\title{
A Simple Statistical Model for Turbulence-Induced Fading in Free-Space Optical Systems
}

\author{
Nestor D. Chatzidiamantis*, Harilaos G. Sandalidis ${ }^{\dagger}$, George K. Karagiannidis*, and Michail Matthaiou ${ }^{\ddagger}$ \\ *Department of Electrical \& Computer Engineering, Aristotle University of Thessaloniki, Thessaloniki, Greece, email: \{nestoras, geokarag\}@auth.gr \\ ${ }^{\dagger}$ Computer Science and Biomedical Informatics, University of Central Greece, Lamia, Greece, e-mail: sandalidis@ucg.gr \\ $\ddagger$ Institute for Circuit Theory and Signal Processing, Technische Universität München (TUM), Munich, Germany, email: matthaiou@nws.ei.tum.de
}

\begin{abstract}
In this paper, we propose the Inverse Gaussian (IG) distribution, as a less complex alternative to Log-normal (LN), to describe turbulence-induced fading in free-space optical (FSO) systems operating in weak turbulence conditions and/or in the presence of a large amount of aperture averaging. By conducting goodness of fit tests, we define the range of values of the scintillation index, where the two distributions approximate each other, with a certain significance level. The efficiency of the new model is pointed out by deriving analytical expressions for the calculation of the bit-error rate of two typical FSO systems; an intensity-modulation/direct detection FSO system with $M$-ary pulse position modulation and a heterodyne FSO system with differential phase shift keying. Numerical examples are provided to clearly illustrate the accuracy of the proposed approach in the weak turbulence regime.
\end{abstract}

\section{INTRODUCTION}

Wireless technology is traditionally associated with radiofrequency (RF) transmission, although transmission via carriers in other parts of the electromagnetic spectrum may be more advantageous for various applications [1]. Operating at unlicensed optical wavelengths, free space optical (FSO) systems offer the potential of broadband communication capacity. As the demand for high speed and tap-proof communications grows, these systems emerge as a cost-effective alternative and/or a complement to radio-frequency (RF) counterparts [2]. Additionally, features such as flexibility, rapid deployment time, high security, and immunity to RF interference have rendered FSO systems appealing as a redundant link for relief efforts, disaster recovery, and military applications.

Despite their significant advantages, there are some major impairments which hamper the widespread deployment of FSO systems. The major performance limiting factor is their high vulnerability to adverse atmospheric conditions. Rain, fog and snow are some deterministic impairments that affect the overall performance. Even in a clear sky, the refractive index of the atmosphere varies, due to inhomogeneities in temperature and pressure changes, and results in atmospheric turbulence. This causes rapid fluctuations at the received optical signal, known as turbulence-induced fading. Such fluctuations increase error rate, thereby severely affecting the reliability of FSO links.

Over the past decades, a number of statistical models have been proposed to describe turbulence-induced fading in different degrees of turbulence severity. One of the most widely used models is the log-normal (LN), which is considered to be very effective in weak turbulence conditions [1], [3]. Moreover, recent studies and experiments have proved that the LN model is also suitable for describing turbulence when the receiver's aperture is larger than the correlation length of irradiance fluctuations (i.e. when aperture averaging takes place) [4] [6]. For these reasons, LN distribution has been extensively employed in the performance analysis of FSO systems [7][9].

Nevertheless, the LN distribution is analytically intractable in the bit-error rate (BER) evaluation of FSO systems. This stems from the fact that the expression for the momentgenerating function (MGF) of the LN distribution cannot be derived in closed form. Hence, no analytical expressions are available in the open technical literature for the BER performance analysis over the LN turbulence model and in this case, numerical methods, such as Gauss-Hermite polynomials [7]- [8], or Monte-Carlo simulations [9], need to be employed.

In this paper, we investigate alternative to LN distributions that efficiently model irradiance fluctuations in weak turbulence conditions or under the presence of aperture averaging, providing simplicity as well. To this end, we propose the Inverse Gaussian (IG) distribution. This statistical model was introduced in [10] as an efficient substitute to LN distribution for describing shadowing effects in RF wireless communications. Moreover, in the context of optical systems, it has been employed for modeling the statistical behavior of avalanche photo diodes receivers [11]. After defining the range of turbulence conditions, where the IG model efficiently approximates LN, we derive analytical expressions for the BER of typical FSO systems, considering the proposed distribution as the turbulence-induced fading model. Specifically, two types of FSO systems are studied: a) an intensity modulation direct detection (IM/DD) FSO system that employs $M$-ary pulse position modulation ( $M \mathrm{PPM}$ ), and, b) a heterodyne FSO system that employs differential phase shift keying (DPSK) modulation and is shot-noise limited. Finally, in order to reveal the accuracy of the proposed approach, we compare the derived analytical results with the BER performance over the LN turbulence model obtained by numerical methods.

The remainder of the paper is organized as follows: In Section II, we present the IG distribution and introduce its basic statistical parameters. In Section III, the IG model is compared with the LN and the range of turbulence conditions, where both models converge with a certain level of signifi- 
cance, is defined. In Section IV, the performance analysis of typical FSO systems operating over the IG channel model is conducted. Concluding remarks can be found in Section V.

\section{Describing TURBUlenCE USING THE IG MODEL}

Let us consider that irradiance fluctuations are modeled by the IG model. The probability density function (pdf) of the IG distributed irradiance, $I$, is given by [12, Eq. (15.4a)]

$$
f_{I}(I)=\sqrt{\frac{\lambda}{2 \pi I^{3}}} e^{-\frac{\lambda(I-\mu)^{2}}{2 \mu^{2} I}}, I>0
$$

where $\mu>0$ is the parameter related to the mean of the fluctuations and $\lambda>0$ is the scale parameter of the distribution. The $n$-th moment of the random variable is given by $[13$, Eq. (2.5)]

$$
\mathrm{E}\left[I^{n}\right]=\mu^{n} \sum_{k=0}^{n-1} \frac{(n-1+k) !}{(n-1+k) !}\left(\frac{\lambda}{2 \mu}\right)^{-k}
$$

where $\mathrm{E}[\cdot]$ denotes expectation.

Without loss of generality, it is assumed that $\mathrm{E}[I]=1$. Using the first moment of (2), it is easily derived that $\mu=1$. Hence, the irradiance scintillation index is obtained by [1]

$$
\sigma_{I}^{2} \triangleq \frac{\mathrm{E}\left[I^{2}\right]}{(\mathrm{E}[I])^{2}}-1=\frac{1}{\lambda}
$$

and as such (1) can be rewritten as

$$
f_{I}(I)=\sqrt{\frac{1}{2 \pi \sigma_{I}^{2} I^{3}}} e^{-\frac{(I-1)^{2}}{2 \sigma_{I}^{2} I}} .
$$

Using the derived parameters, the cumulative distribution function (cdf) of the IG model can be written as [13, Eq. (2.14)]

$$
F_{I}(I)=1-Q\left(\frac{I-1}{\sqrt{I} \sigma_{I}}\right)+e^{\frac{2}{\sigma_{I}^{2}}} Q\left(\frac{I+1}{\sqrt{I} \sigma_{I}}\right)
$$

where $Q(\cdot)$ is the Gaussian $Q$-function, defined as $Q(x)=$ $\frac{1}{\sqrt{2 \pi}} \int_{x}^{\infty} e^{-\frac{t^{2}}{2}} d t$ and related to the complementary error function $\operatorname{erfc}(\cdot)$ by $Q(x)=\frac{1}{2} \operatorname{erfc}\left(\frac{x}{\sqrt{2}}\right)$.

\section{COMPARISON WITH THE LN MODEL}

In this section, we investigate the validity of the IG approximation to the $\mathrm{LN}$ distribution using statistical tools and arguments. Specifically, we employ Kolmogorov-Smirnov (KS) goodness of fit statistical tests in order to measure the difference between the two models. These tests measure the maximum value of the absolute difference between the empirical cdf of IG distributed data, $F_{x}(\cdot)$, and the cdf of the LN model [14, pp. 272-273]. Hence, the KS test statistic is defined as

$$
T \triangleq \max \left|F_{x}(x)-F_{y}(x)\right|
$$

where $F_{y}(x)$ is the cdf of the LN model given by [5]

$$
F_{y}(x)=1-Q\left(\frac{\ln \left(x \sqrt{\sigma_{I}^{2}+1}\right)}{\sqrt{\ln \left(\sigma_{I}^{2}+1\right)}}\right) .
$$

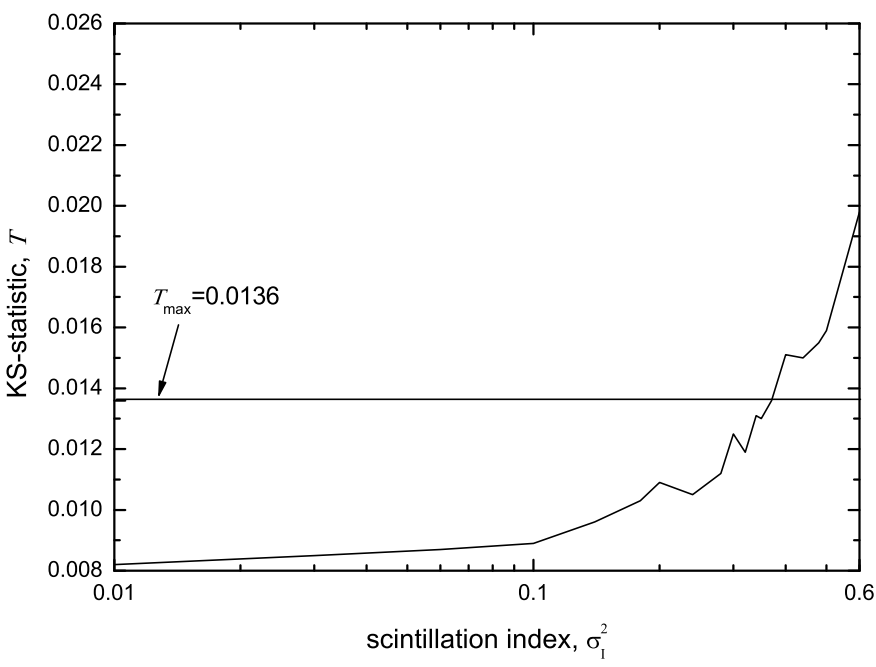

Fig. 1. Hypothesis testing distribution using the KS goodness-of-fit test for the IG to approximate the LN distribution with a 5\% significance level.

Definition 1: We define $\boldsymbol{H}_{0}$ as the null hypothesis under which the IG distributed data belongs to the cdf of the LN distribution $F_{y}(\cdot)$.

To test $\boldsymbol{H}_{0}$, the KS goodness-of-fit test compares the test statistic $T$ to a critical level $T_{\max }$ for a given significance level $\alpha$. Any hypothesis for which $T>T_{\max }$, is rejected with significance $1-\alpha$, while any hypothesis for which $T<T_{\max }$ is accepted with the same level of significance.

Figure 1 depicts the KS test statistic for different values of scintillation index, $\sigma_{I}^{2}$, which correspond to different degrees of turbulence severity. The presented test results have been obtained by averaging the results of 60 simulation runs, each for at least $10^{4}$ samples of IG distributed data. The critical value $T_{\max }$, used for comparison reasons, is equal to $T_{\max }=0.0136$ which corresponds to a significance level of $\alpha=5 \%$ [14, Eq. (9-73)]. As it is clearly illustrated in the figure, the hypothesis $\boldsymbol{H}_{0}$ is accepted with $95 \%$ significance until $\sigma_{I}^{2}$ reaches the value of 0.35 . Hence, the IG model can be considered as a good alternative of the LN model in this turbulence regime (defined as $\sigma_{I}^{2}$ less than 0.35), which describes irradiance fluctuations in weak turbulence conditions [1].

\section{Performance Analysis}

In this section, we point out the simplicity of the IG channel model by providing analytical expressions for the performance evaluation of typical FSO systems operating over weak turbulence conditions or in the presence of a large amount of aperture averaging effects.

\section{A. MPPM IM/DD System}

We consider an IM/DD FSO system that employs MPPM. For $M P P M$, the symbol period $T_{S}$ is divided into $M$ equal non-overlapping time slots of duration $T_{p}=\frac{T_{S}}{M}$ and a digital message, comprised of $\log _{2} M$ bits, is sent by pulsing the laser's intensity in one of these slots. At the receiver, the 
Poisson photon-counting detection process is assumed [9] and the received signal is a vector of $M$ Poisson observations that correspond to the photon counts at each time slot. The average number of photoelectrons per slot when a pulse is transmitted, is denoted by $n_{s}$ and is given by

$$
n_{s}=\frac{\eta P_{r} T_{p}}{h \nu}
$$

where $\eta$ represents the photo-detector's optical-to-electrical efficiency, $h$ is the Planck's constant in Joules $/ \mathrm{Hz}, \nu$ is the frequency of the optical signal in Hertz and $P_{r}$ is the total power incident on the photodetector at the time slot. Similarly, the average number of photoelectrons per slot due to background noise, $n_{b}$, is defined as

$$
n_{b}=\frac{\eta P_{b} T_{p}}{h \nu}
$$

where $P_{b}$ is the power incident on the photodetector due to background noise. Hence, the probability mass function of the received signal at the photodetector is given by

$$
\begin{aligned}
\operatorname{Pr}\{\boldsymbol{r} \mid \boldsymbol{s}, I\} & =\prod_{m=1}^{M} \operatorname{Pr}\left\{r_{m} \mid s_{m}, I\right\} \\
& =\prod_{m=1}^{M} \frac{\left(n_{s} s_{m} I+n_{b}\right)^{r_{m}} e^{-\left(n_{s} s_{m} I+n_{b}\right)}}{r_{m} !}
\end{aligned}
$$

where $s_{m} \in[0,1]$, depending on whether a pulse is transmitted in time slot $m$ and $I$ is the fading coefficient, which remains constant during the symbol period ${ }^{1}$.

The conditioned on $I$ symbol error probability (SEP) can be obtained by [16] as

$$
\begin{aligned}
P_{s}(e \mid I) & =1-\frac{1}{M} \sum_{k=0}^{\infty}\left(\frac{n_{s}}{n_{b}} I+1\right)^{k} e^{-n_{s} I} \\
& \times\left(P_{o}(k)^{M}-P_{o}(k-1)^{M}\right)
\end{aligned}
$$

where

$$
P_{o}(k)=\sum_{i=0}^{k} \frac{n_{b}^{i} e^{-n_{b}}}{i !}=\frac{\Gamma\left(k+1, n_{b}\right)}{\Gamma(k+1)}
$$

with $\Gamma(\cdot)$ and $\Gamma(\cdot, \cdot)$ denoting the Gamma [22, Eq. (8.310)] and the upper incomplete Gamma [22, Eq. (8.352.2)] functions, respectively. The average SEP is derived by averaging (11) over the pdf of turbulence, i.e.

$$
\begin{aligned}
P_{s}(e) & =\int_{0}^{\infty} P(e \mid I) f_{I}(I) d I \\
& =1-\frac{1}{M} \sum_{k=0}^{\infty}\left(P_{o}(k)^{M}-P_{o}(k-1)^{M}\right) g(k)
\end{aligned}
$$

where

$$
g(k)=\int_{0}^{\infty}\left(\frac{n_{s}}{n_{b}} I+1\right)^{k} e^{-n_{s} I} f_{I}(I) d I .
$$

\footnotetext{
${ }^{1}$ Taking into consideration the coherence time of the FSO channel and the signaling rates of interest, this assumption is quite realistic according to [15].
}

For the IG distributed turbulence-induced fading model, (1) is substituted into (14) and yields

$g_{\text {Inv }}(k)=\sqrt{\frac{1}{2 \pi \sigma_{I}^{2}}} \int_{0}^{\infty} I^{-\frac{3}{2}}\left(\frac{n_{s}}{n_{b}} I+1\right)^{k} e^{-\left(n_{s} I+\frac{(I-1)^{2}}{2 \sigma_{I}^{2}}\right)} d I$

The above equation contains an integral of the form

$$
\int_{0}^{\infty} x^{-r}(a x+1)^{k} e^{-\left(b x+\frac{c}{x}\right)} d x
$$

which can be solved in closed form as in the Appendix. Hence, (15) can be rewritten as

$$
\begin{aligned}
g_{\text {Inv }}(k) & =\sum_{j=0}^{k}\left(\begin{array}{c}
k \\
j
\end{array}\right) \frac{e^{\frac{1}{\sigma_{I}^{2}}}}{\sigma_{I}}\left(\frac{n_{s}}{n_{b}}\right)^{j} \\
& \times \sqrt{\frac{2}{\pi}}\left(1+2 n_{s} \sigma_{I}^{2}\right)^{\frac{1}{4}-\frac{j}{2}} \\
& \times K_{j-\frac{1}{2}}\left(\frac{\sqrt{1+2 n_{s} \sigma_{I}^{2}}}{\sigma_{I}^{2}}\right) .
\end{aligned}
$$

By substituting (17) in (13), the SEP can be analytically evaluated and hence, the BER can be obtained by [9]

$$
P_{b}(e)=\frac{M}{2(M-1)} P_{s}(e) .
$$

It should be noted that no closed-form solution is available for (14), when the LN turbulence model is considered. In this case, Gauss-Hermite polynomials are employed, according to [8]

$$
\begin{aligned}
g_{L n}(k) & =\frac{1}{k ! \sqrt{\pi}} \sum_{n=1}^{n_{p}} H_{x_{n}}\left(n_{b}+e^{\left(\mu+\sqrt{2} \sigma x_{n}\right)}\right)^{k} \\
& \times e^{-\left(n_{b}+e^{\left(\mu+\sqrt{2} \sigma x_{n}\right)}\right)}
\end{aligned}
$$

where $x_{n}$ are the zeros, $H_{x_{n}}$ are the weight factors of the $N_{p}$-order Hermite polynomial given by [17, Table (25.10)]. Furthermore, $\sigma=\sqrt{\ln \left(\sigma_{I}^{2}+1\right)}$ and $\mu=-0.5 \ln \left(\sigma_{I}^{2}+1\right)$.

Figures 2 and 3 illustrate the average BER of a MPPM FSO system operating in weak turbulence conditions, when different levels of background energy, $E_{b a c k}=P_{b} T_{p}$, are assumed. In each figure, the performance of the IG model, derived by (17) and (18), is depicted as a function of the energy per bit, $E_{b}=\frac{P_{r} T_{p}}{\log _{2} M}$, and is plotted in comparison with the performance of the LN turbulence model, for different values of scintillation index and modulation order. As it is clearly illustrated, the approximative analytical results are very close to the performance of the $\mathrm{LN}$ model, derived by numerical integrations. Specifically, they lie within $2 \mathrm{dBJs}$ for practical values of BER in all turbulence conditions considered (scintillation index less that 0.35 ). Moreover, it can easily be observed that the performance of the IG model acts as a tight lower bound, whose tightness looses as the scintillation index increases, irrespective of the assumed modulation order and/or level of background energy. 


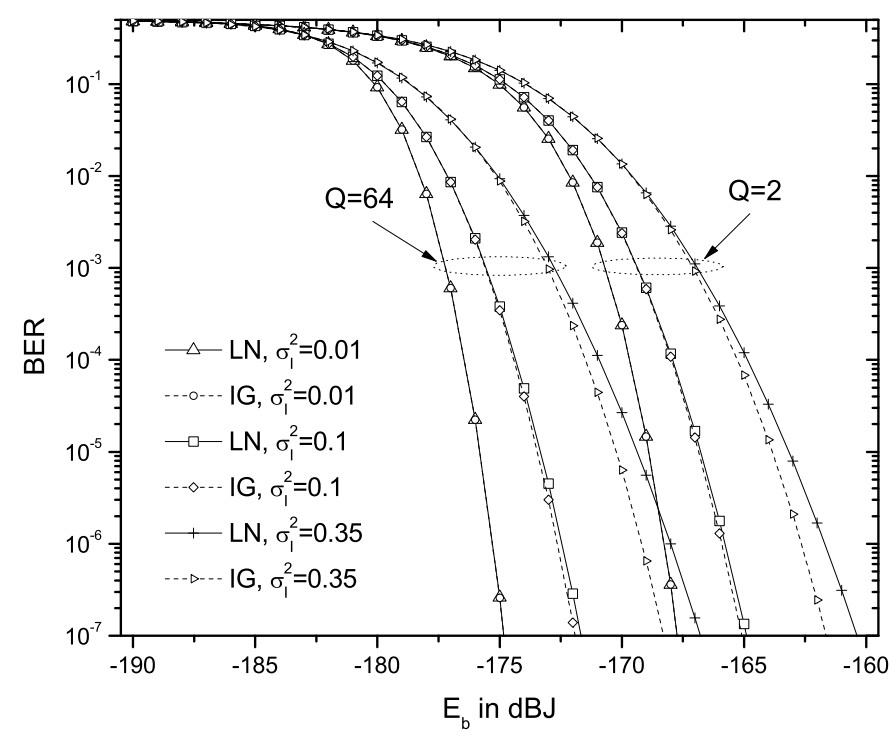

Fig. 2. BER performance of $Q P P M$ systems for different scintillation indices and modulation orders, assuming background energy $E_{\text {back }}=-170 \mathrm{dBJ}$.

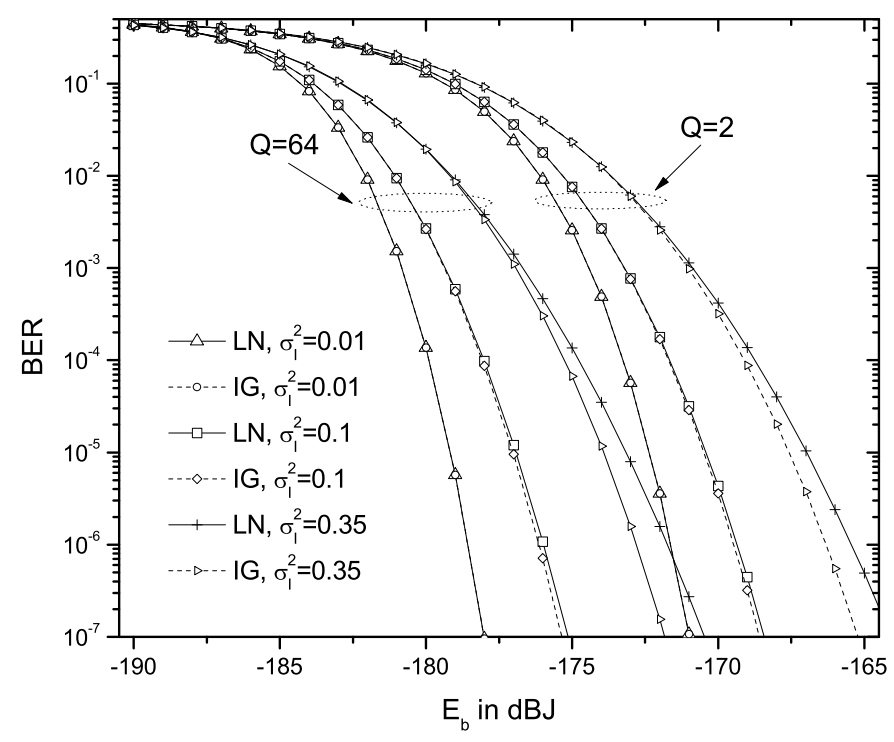

Fig. 3. BER performance of $Q P P M$ systems for different scintillation indices and modulation orders, assuming background energy $E_{b a c k}=-180 \mathrm{dBJ}$.

\section{B. Heterodyne DPSK System}

For a coherent shot noise limited DPSK system, the conditional BER is obtained by [18]

$$
P(e \mid I)=\frac{1}{2} e^{-\bar{\gamma} I}
$$

where

$$
\bar{\gamma}=\frac{\eta A T_{s}}{h \nu}
$$

denotes the average SNR, without turbulence. In the above equation, $A$ is the detector area in square meters and $T_{s}$ is the DPSK symbol duration in seconds. Note that we follow the same assumptions as in [18] and we particularly consider that irradiance remains constant over a symbol interval. That is, we assume that the duration of $T_{s}$ is smaller than the coherence time of the channel, thus having a constant amplitude scenario for the received signal [19].

For the slow-fading environment under consideration, the average BER, $P(e)$, can be obtained by averaging (20) over the pdf of $I$ [20], i.e.,

$$
P(e)=\int_{0}^{\infty} P(e \mid I) f_{I}(I) d I
$$

where $f_{I}(I)$ follows the IG distribution. Since the definition of the MGF is given in closed form as [13, Eq. (2.14)]

$$
\begin{aligned}
M_{\text {Inv }}(s) & =\int_{0}^{\infty} f_{I}(I) e^{s I} d I \\
& =e^{\frac{1}{\sigma_{I}^{2}}\left(1-\sqrt{1-\frac{2}{\sigma_{I}^{2}} s}\right)}
\end{aligned}
$$

the average BER is derived analytically as

$$
P(e)=\frac{1}{2} M_{\text {Inv }}(-\bar{\gamma})
$$

Note that the MGF of the LN distribution is given approximately in terms of Gauss-Hermite polynomials as [21, Eq. (2.54)]

$$
M_{L n}(s) \simeq \frac{1}{\sqrt{\pi}} \sum_{n=1}^{N_{p}} H_{x_{n}} e^{s 10^{\left(\sqrt{2} \sigma x_{n}+\mu\right) / 10}}
$$

where $x_{n}, H_{x_{n}}, N_{p}, \sigma$ and $\mu$ were defined earlier.

Figure 4 illustrates the average BER performance of a heterodyne DPSK FSO system assuming weak turbulence conditions. Analytical results, obtained by (23) and (24), are plotted in comparison with the performance of the LN turbulence model for scintillation indices less than 0.35. As it is clearly depicted, the performance of the IG model is very close to the performance of the LN model. Specifically, it lies within $2 \mathrm{~dB}$ for the BER values of interest. Moreover, it is observed that the performance of the IG distribution acts as a tight lower bound; the lower the scintillation index the tighter the bound.

\section{Conclusions}

In this paper, we introduced the IG statistical model as a simpler alternative to the $\mathrm{LN}$ distribution. The new pdf can efficiently describe turbulence-induced fading in FSO systems in weak turbulence conditions or in the presence of large amount of aperture averaging effects. After comparing the two models using KS goodness-of-fit tests, the range of the scintillation indices being less than 0.35 was defined, in which IG approximates LN with 5\% significance level. Moreover, the simplicity of the IG was pointed out by deriving analytical BER expressions for some typical FSO systems (IM/DD with MPPM and heterodyne DPSK, respectively). Comparisons between the BER performance metrics over the IG and the more complicated LN model, for which no analytical results are available, were also provided to illustrate the accuracy of the proposed approach. 


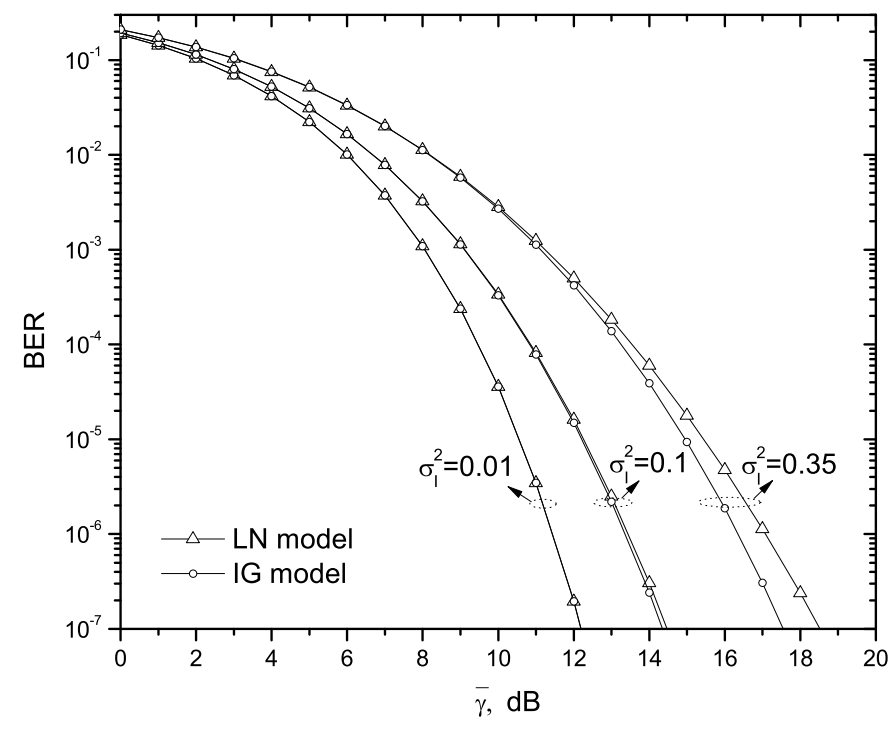

Fig. 4. BER performance of DPSK systems for different scintillation indices.

\section{APPENDIX}

This Appendix provides an analytical solution for the integral of the form

$$
f(k)=\int_{0}^{\infty} x^{-r}(a x+1)^{k} e^{-\left(b x+\frac{c}{x}\right)} d x
$$

where the variable $k$ is a positive integer. The above integral is difficult to be solved directly due to the term $(a x+1)^{k}$. However, taking into cosideration that $k \in \mathbb{Z}^{+}$, this term can be expanded according to the binomial theorem [22, Eq. (1.111)] as

$$
(a x+1)^{k}=\sum_{j=0}^{k}\left(\begin{array}{c}
k \\
j
\end{array}\right)(a x)^{j}
$$

and hence (26) can be written as

$$
f(k)=\sum_{j=0}^{k}\left(\begin{array}{c}
k \\
j
\end{array}\right) a^{j} \int_{0}^{\infty} x^{j-r+1-1} e^{-\left(b x+\frac{c}{x}\right)} d x
$$

Using [22, Eq. (3.471.9)], a closed form solution is obtained as

$$
f(k)=\sum_{j=0}^{k}\left(\begin{array}{c}
k \\
j
\end{array}\right) a^{j} 2\left(\frac{c}{b}\right)^{\frac{1-r+j}{2}} K_{1-r+j}(2 \sqrt{b c})
$$

where $K_{\nu}(\cdot)$ is the $\nu$-th order modified Bessel function of the second kind [22, Eq. (8.432.9)].

\section{REFERENCES}

[1] L. Andrews, R. L. Philips, and C. Y. Hopen, Laser Beam Scintillation with Applications. SPIE Press, 2001.

[2] H. Willebrand and B. S. Ghuman, Free Space Optics: Enabling Optical Connectivity in Todays Networks. Sams Publishing, 2002.

[3] G. Parry, "Measurement of atmospheric turbulence induced intensity fluctuations in a laser beam," J. Modern Opt., vol. 28, no. 5, pp. 715728, May 1981.

[4] F. S. Vetelino, C. Young, L. Andrews, and J. Recolons, "Aperture averaging effects on the probability density of irradiance fluctuations in moderate-to strong turbulence," Appl. Opt., vol. 46, no. 11, Apr. 2007.

[5] D. Giggenbach and H. Henniger, "Fading-loss assessment in atmospheric free-space optical communication links with on-off keying," Opt. Engineer, vol. 47, no. 4, Apr. 2008.

[6] N. Perlot and D. Fritzsche, "Aperture-averaging- theory and measurements," in Proc. SPIE 5338, vol. 5338, San Jose, CA, Jul. 2004, pp. $233-242$.

[7] S. M. Navidpour, M. Uysal, and M. Kavehrad, "BER performance of free-space optical transmission with spatial diversity," IEEE Trans. Wireless Commun., vol. 6, no. 8, pp. 2813-2819, Aug. 2007.

[8] W. Gappmair and S. S. Muhammad, "Error performance of terrestrial FSO links modelled as PPM/Poisson channels in turbulent atmosphere," IEE Electron. Lett., vol. 43, no. 5, pp. 63-64, Mar. 2007.

[9] S. G. Wilson, M. Brandt-Pearce, Q. Qao, and J. H. Leveque, "Free-space optical MIMO transmission with $Q$-ary PPM," IEEE Trans. Commun., vol. 53, no. 8, pp. 1402-1411, Aug. 2005.

[10] Karmeshu and R. Agrawal, "On efficacy of Rayleigh-inverse Gaussian distribution over K-distribution for wireless fading channels," Wireless Commun. Mobile Comput., vol. 7, no. 1, pp. 1-7, Jan. 2007.

[11] K. R. Baker, "On the WMC density as an inverse Gaussian probability density," IEEE Trans. Commun., vol. 44, no. 1, pp. 15-17, Jan. 1996.

[12] S. Kotz, Continuous univariate distributions, 2nd ed. Wiley, 1994, vol. 1.

[13] R. S. Chhikara and J. L. Folks, The Inverse Gaussian distribution: Theory, methodology, and applications. New York: M. Dekker, 1989.

[14] A. Papoulis, Probability, Random Variables, and Stochastic Processes, 3rd ed. McGraw-Hill, 1991.

[15] D. J. T. Heatley, D. R. Wisely, I. Neild, and P. Cochrane, "Optical wireless: The story so far," IEEE Commun. Mag., vol. 36, no. 2, pp. 72-74, Dec. 1998.

[16] J. Hamkins, "Accurate computation of the performance of M-ary orthogonal signaling on a discrete memoryless channel," IEEE Trans. Commun., vol. 52, no. 11, pp. 1844-1845, Nov. 2004.

[17] M. Abramovitz and I. A. Stegun, Handbook of Mathematical Functions with Formulas, Graphs, and Mathematical Tables, 9th ed. New York: Dover, 1972.

[18] K. Kiasaleh, "Performance of coherent DPSK free-space optical communication systems in K-distributed turbulence," IEEE Trans. Commun., vol. 54, no. 4, pp. 604-607, Apr. 2006.

[19] H. G. Sandalidis, T. A. Tsiftsis, and G. K. Karagiannidis, "Optical wireless communications with heterodyne detection over turbulence channels with pointing errors," IEEE/OSA J. Lightw. Techn., vol. 27, no. 12, pp. 4440-4445, Oct. 2009.

[20] T. A. Tsiftsis, "Performance of heterodyne wireless optical communication systems over gamma-gamma atmospheric turbulence channels," IET Electron. Lett., vol. 44, no. 5, pp. 372-373, Feb. 2008.

[21] M. K. Simon and M. -S. Alouini, Digital Communication over Fading Channels, 2nd ed. New York: Wiley, 2005.

[22] I. S. Gradshteyn and I. M. Ryzhik, Table of Integrals, Series, and Products, 7th ed. New York: Academic, 2007. 